\title{
Critical Behavior of the Chayes-Machta-Swendsen-Wang Dynamics
}

\author{
Youjin Deng, ${ }^{1}$ Timothy M. Garoni, ${ }^{1}$ Jonathan Machta, ${ }^{2}$ Giovanni Ossola, ${ }^{3}$ Marco Polin,,${ }^{1}$ and Alan D. Sokal ${ }^{1,4}$ \\ ${ }^{1}$ Department of Physics, New York University, 4 Washington Place, New York, New York 10003, USA \\ ${ }^{2}$ Department of Physics, University of Massachusetts, Amherst, Massachusetts 01003, USA \\ ${ }^{3}$ Institute of Nuclear Physics, NCSR "Demokritos," 15310 Athens, Greece \\ ${ }^{4}$ Department of Mathematics, University College London, London WC1E 6BT, United Kingdom
}

(Received 18 May 2007; published 31 July 2007)

\begin{abstract}
We study the dynamic critical behavior of the Chayes-Machta dynamics for the Fortuin-Kasteleyn random-cluster model, which generalizes the Swendsen-Wang dynamics for the $q$-state Potts model to noninteger $q$, in two and three spatial dimensions, by Monte Carlo simulation. We show that the Li-Sokal bound $z \geq \alpha / \nu$ is close to but probably not sharp in $d=2$ and is far from sharp in $d=3$, for all $q$. The conjecture $z \geq \beta / \nu$ is false (for some values of $q$ ) in both $d=2$ and $d=3$.
\end{abstract}

DOI: 10.1103/PhysRevLett.99.055701

PACS numbers: 64.60.Ht, 05.10.Ln, 05.50.+q, 05.70.Jk

Monte Carlo simulations in statistical mechanics [1] and quantum field theory [2] typically suffer from critical slowing-down [3,4]: the autocorrelation (relaxation) time $\tau$ diverges as the critical point is approached, most often like $\tau \sim \xi^{z}$, where $\xi$ is the spatial correlation length and $z$ is a dynamic critical exponent. For local algorithms, one usually has $z \approx 2$. This effect severely limits the efficiency of Monte Carlo studies of critical phenomena in statistical mechanics and of the continuum limit in quantum field theory.

An important advance was made in 1987 with the invention of the Swendsen-Wang (SW) cluster algorithm [5] for simulating the $q$-state ferromagnetic Potts model $[6,7]$ at positive integer $q$. The SW algorithm is based on passing back and forth between the Potts spin representation and the Fortuin-Kasteleyn (FK) bond representation [8,9]. This algorithm does not eliminate critical slowing-down, but it radically reduces it compared to local algorithms. Much effort has therefore been devoted, for both theoretical and practical reasons, to understanding the dynamic critical behavior of the SW algorithm as a function of the spatial dimension $d$ and the number $q$ of Potts spin states [10]. Unfortunately, it is very difficult to develop a physical understanding from the small number of "data points" at our disposal: second-order transitions occur only for $(d, q)=(2,2),(2,3),(2,4),(3,2)$, and $(4,2)[11]$.

A further advance was made in 1998 by Chayes and Machta (CM) [12], who devised a cluster algorithm for simulating the FK random-cluster model $[8,13]$ - which provides a natural extension of the Potts model to noninteger $q$-at any real $q \geq 1$. The CM algorithm generalizes the SW algorithm and in fact reduces to (a slight variant of) it when $q$ is an integer. By using the $\mathrm{CM}$ algorithm, we can study the dynamic critical behavior of the SW-CM dynamic universality class as a function of the continuous variable $q$ throughout the range $1 \leq q \leq$ $q_{c}(\mathcal{L})$, where $q_{c}(\mathcal{L})$ is the maximum $q$ for which the transition is second-order on the lattice $\mathcal{L}$ [14]. This vastly enhances our ability to make theoretical sense of the numerical results.

In this Letter, we report detailed measurements of the dynamic critical behavior of the CM algorithm for twodimensional random-cluster models with $1 \leq q \leq 4$ [15] and for three-dimensional models with $q=1.5,1.8,2$, and 2.2 [16]. Among other things, we find strong evidence against the conjecture $z \geq \beta / \nu$ recently proposed by two of us [10], which had seemed plausible from the data for integer $q$.

The $F K$ random-cluster model with parameter $q>0$ is defined on any finite graph $G=(V, E)$ by the partition function

$$
Z=\sum_{A \subseteq E} q^{k(A)} \prod_{e \in A} v_{e}
$$

where $A$ is the set of "occupied bonds" and $k(A)$ is the number of connected components ("clusters") in the graph $(V, A)$; here $\left\{v_{e}\right\}$ are non-negative edge weights. For $q=1$ this reduces to independent bond percolation [17] with occupation probabilities $p_{e}=v_{e} /\left(1+v_{e}\right)$; for integer $q \geq 1$ it provides a graphical representation of the $q$-state ferromagnetic Potts model with nearest-neighbor couplings $\left\{J_{e}\right\}$, where $v_{e}=e^{\beta J_{e}}-1$.

It is convenient to consider a generalized randomcluster (RC) model [16,18]

$$
Z=\sum_{A \subseteq E}\left(\prod_{e \in A} v_{e}\right)\left(\prod_{i=1}^{k} W\left(H_{i}\right)\right)
$$

where $H_{1}, \ldots, H_{k}$ are the connected components of the graph $(V, A)$, and $\{W(H)\}$ are non-negative weights associated to the connected subgraphs $H$ of $G$. The model (2) reduces to the FK model (1) if $W(H)=q$ for all $H$; other special cases include an FK representation for the Potts model in a magnetic field [19] and various loop models [18]. 
Now let $m$ be a positive integer, and let us decompose each weight $W(H)$ into $m$ non-negative pieces, any way we like: $W(H)=\sum_{\alpha=1}^{m} W_{\alpha}(H)$. The first step of our generalized Chayes-Machta algorithm, given a bond configuration $A$, is to choose, independently for each connected component $H_{i}$, a "color" $\alpha \in\{1, \ldots, m\}$ with probabilities $W_{\alpha}\left(H_{i}\right) / W\left(H_{i}\right)$; this color is then assigned to all of the vertices of $H_{i}$. The vertex set $V$ is thus partitioned as $V=$ $\bigcup_{\alpha=1}^{m} V_{\alpha}$. It is not hard to see that, conditioning on this decomposition, the bond configuration is nothing other than a generalized RC model with weights $\left\{W_{\alpha}(H)\right\}$ on the induced subgraph $G\left[V_{\alpha}\right]$, independently for each $\alpha$.

We now have the right to update these generalized RC models by any valid Monte Carlo algorithm. One valid update is "do nothing"; this corresponds to the "inactive" colors of Chayes and Machta [12]. Of course, we must also include at least one nontrivial update. The basic idea is to have at least one color for which the weights $W_{\alpha}(H)$ are "easy" to simulate. In particular, when $W(H)=q$ for all $H$ (the standard FK random-cluster model), we can take $W_{\alpha}(H)=1$ for one or more colors $\alpha$ (the so-called "active" colors); the corresponding model on $G\left[V_{\alpha}\right]$ is then independent bond percolation, which can be trivially updated. Since we must have $W_{\alpha}(H) \leq W(H)$, this works whenever $q \geq 1$. More generally, if $q \geq k$, then we can have $k$ active colors. If $q$ is an integer and we take $k=q$, we recover the standard SW algorithm.

We used the CM algorithm to simulate the randomcluster model in dimensions $d=2,3$ on hypercubic lattices of size $L^{d}$ with periodic boundary conditions. We measured the "energylike" observables $\mathcal{N}=$ \# of occupied bonds and $\mathcal{E}^{\prime}=\#$ of nearest-neighbor pairs belonging to the same cluster, the cluster-size moments $\mathcal{S}_{m}=\sum|C|^{m}$ for various values of $m$, where $|C|$ is the number of sites in the cluster $C$, and the size $\mathcal{C}_{i}$ of the $i$ th-largest cluster for $i=1,2,3$.

For any observable $\mathcal{O}$, let $\rho_{\mathcal{O}}(t)$ be its normalized autocorrelation function. Then define the exponential autocorrelation time

$$
\tau_{\exp , \mathcal{O}}=\limsup _{t \rightarrow \pm \infty} \frac{|t|}{-\log \left|\rho_{\mathcal{O}}(t)\right|}
$$

and the integrated autocorrelation time

$$
\tau_{\mathrm{int}, \mathcal{O}}=\frac{1}{2} \sum_{t=-\infty}^{\infty} \rho_{\mathcal{O}}(t)
$$

Typically, all observables $\mathcal{O}$ (except those that, for symmetry reasons, are "orthogonal" to the slowest mode) have the same value $\tau_{\text {exp } \mathcal{O}}=\tau_{\text {exp }}$. However, they may have very different amplitudes of "overlap" with this slowest mode; in particular, they may have very different values of the integrated autocorrelation time, which controls the efficiency of Monte Carlo simulations [4]. We define dynamic critical exponents $z_{\exp }$ and $z_{\text {int, } \mathcal{O}}$ by $\tau_{\exp } \sim \xi^{z_{\exp }}$ and

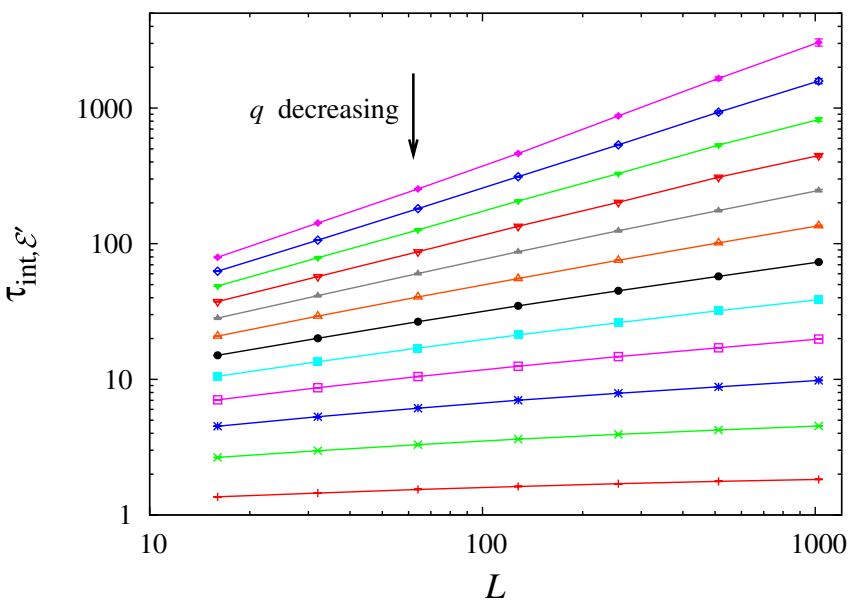

FIG. 1 (color online). Integrated autocorrelation times $\tau_{\text {int, } \mathcal{E}^{\prime}}$ versus lattice size $L$ for the critical two-dimensional randomcluster model at $1.25 \leq q \leq 4$, simulated using the ChayesMachta algorithm with $k=1$.

$\tau_{\text {int, } \mathcal{O}} \sim \xi^{z_{\text {int },} \mathcal{O}}$. On a finite lattice at criticality, $\xi$ can here be replaced by $L$.

We began by performing simulations on the square lattice $(d=2)$ at the exact critical point $v_{c}(q)=\sqrt{q}$ [20] for $1.25 \leq q \leq 4$ in steps of 0.25 and lattice sizes $16 \leq$ $L \leq 1024$, using all positive integer values of $k \leq q$. We estimated the integrated autocorrelation times $\tau_{\text {int, } \mathcal{O}}$ by the automatic windowing method described in Refs. [10,21]. The complete set of runs used approximately $14.8 \mathrm{yr}$ CPU time on a $1266 \mathrm{MHz}$ Pentium III Tualatin processor.

The autocorrelation functions of $\mathcal{N}, \mathcal{E}^{\prime}$, and $\mathcal{S}_{m}$ are in all cases very close to a pure exponential. In Fig. 1, we plot $\tau_{\text {int, } \mathcal{E}^{\prime}}($ for $k=1$ ) versus $L$, and, in Table I, we report the estimated dynamic critical exponents $z_{\text {int, } \mathcal{E}^{\prime}}$. Our data also

TABLE I. Dynamic critical exponents $z_{\text {int, } \mathcal{E}^{\prime}}$ for the twodimensional random-cluster model as a function of $q$, with preferred fit and minimum $L$ value used in the fit. Error bars are 1 standard deviation, statistical error only. The exact exponents $\alpha / \nu$ and $\beta / \nu$ are shown for comparison [22].

\begin{tabular}{ccrlcc}
\hline \hline$q$ & Fit & $L_{\text {min }}$ & $z_{\text {int } \mathcal{E}^{\prime}}$ & $\alpha / \nu$ & $\beta / \nu$ \\
\hline 1.00 & Exact & $\ldots$ & 0 & -0.5000 & 0.1042 \\
1.25 & $A+B L^{-p}$ & 128 & 0 & -0.3553 & 0.1112 \\
1.50 & $A+B L^{-p}$ & 32 & 0 & -0.2266 & 0.1168 \\
1.75 & $A L^{z}+B$ & 16 & $0.06(1)$ & -0.1093 & 0.1213 \\
2.00 & $A L^{z}+B$ & 32 & $0.14(1)$ & $0(\log )$ & 0.1250 \\
2.25 & $A L^{z}+B$ & 32 & $0.24(1)$ & 0.1036 & 0.1280 \\
2.50 & $A L^{z}+B$ & 32 & $0.31(1)$ & 0.2036 & 0.1303 \\
2.75 & $A L^{z}+B$ & 16 & $0.40(2)$ & 0.3017 & 0.1321 \\
3.00 & $A L^{z}+B$ & 32 & $0.49(1)$ & 0.4000 & 0.1333 \\
3.25 & $A L^{z}+B$ & 64 & $0.57(1)$ & 0.5013 & 0.1339 \\
3.50 & $A L^{z}$ & 16 & $0.69(1)$ & 0.6101 & 0.1338 \\
3.75 & $A L^{z}$ & 32 & $0.78(1)$ & 0.7376 & 0.1324 \\
4.00 & $A L^{z}+B$ & 32 & $0.93(2)$ & 1.0000 & 0.1250 \\
\hline \hline
\end{tabular}




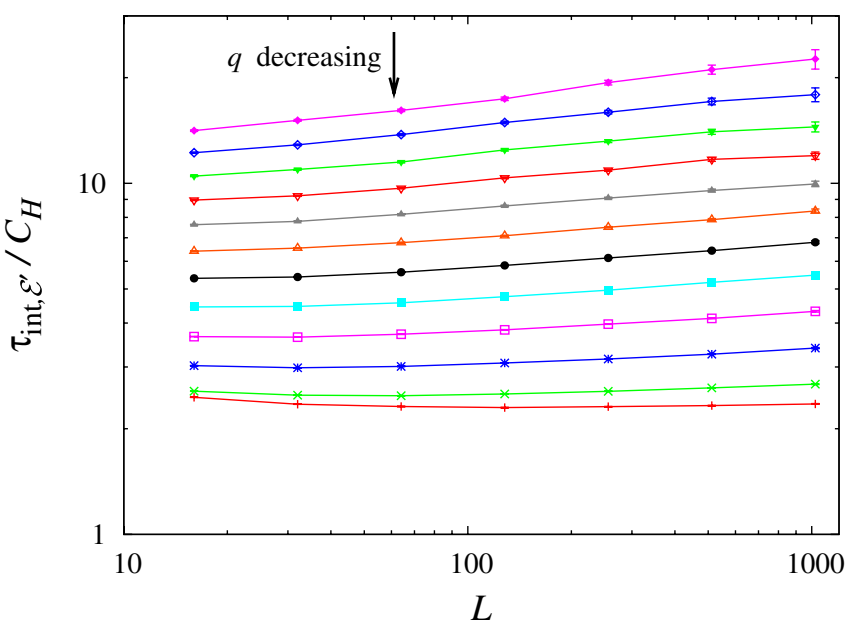

FIG. 2 (color online). Integrated autocorrelation times $\tau_{\text {int, } \mathcal{E}^{\prime}}$ divided by specific heat $C_{H}$, versus lattice size $L$, for the critical two-dimensional random-cluster model at $1.25 \leq q \leq 4$, simulated using the Chayes-Machta algorithm with $k=1$.

show that, as expected, the exponents are independent of $k$, and we have roughly $\tau \propto 1 / k$.

Since the Li-Sokal bound $\tau_{\text {int, } \mathcal{N}}, \tau_{\text {int, } \mathcal{E}^{\prime}} \geq$ const $\times C_{H}$ and hence $z_{\text {int, }, \mathcal{N}}, z_{\text {int }, \mathcal{E}^{\prime}} \geq \alpha / \nu$, originally proven [23] for the Swendsen-Wang algorithm, can also be proven [16] for the Chayes-Machta algorithm (at least for $\mathcal{N}$ ), it is of interest to analyze its possible sharpness [24]. In Fig. 2, we plot $\tau_{\text {int, } \mathcal{E}^{\prime}} / C_{H}$ versus $L$, in an attempt to determine whether this ratio is bounded or not as $L \rightarrow \infty$. The results are far from clear, but our best guess is that $\tau_{\mathrm{int}, \mathcal{E}^{\prime}} / C_{H}$ diverges as $L \rightarrow \infty$, either as a small power or as a logarithm. However, the precise behavior needs to be explored by simulations at larger $L$.

On the other hand, Ossola and Sokal [10] recently conjectured, on the basis of the "data points" $(d, q)=(2,2)$, $(2,3),(2,4),(3,2)$, and $(4,2)$, that $z_{\mathrm{int}, \mathcal{E}^{\prime}} \geq \beta / \nu$, and they even speculated that we might have the equality $z_{\text {int, } \mathcal{E}^{\prime}}=$ $\max (\alpha / \nu, \beta / \nu)$. The data for noninteger $q$ now shed light on this conjecture: for $q=1.25,1.5$ there is modest evidence (and for $q=1.75$ there is weak evidence) that $z_{\text {int, } \mathcal{E}^{\prime}}<\beta / \nu$, i.e., that even the weak form of the OssolaSokal conjecture is false.

We next performed simulations on the simple-cubic lattice $(d=3)$ for $q=1.5,1.8,2.2$ (see also [10] for $q=2$ ) and lattice sizes $4 \leq L \leq 256$, using $k=$ the largest integer $\leq q$. We located the critical point by a finite-size-scaling analysis using the ratio $R=\left\langle\mathcal{S}_{4}\right\rangle /\left\langle\mathcal{S}_{2}^{2}\right\rangle$, as in Ref. [25]. The complete set of runs used approximately $21.5 \mathrm{yr}$ CPU time on a $3.2 \mathrm{GHz}$ Xeon EM64T processor.

The autocorrelation functions of $\mathcal{N}, \mathcal{E}^{\prime}$, and $\mathcal{S}_{m}$ are again very close to a pure exponential. In Fig. 3 , we plot $k \tau_{\text {int, } \mathcal{E}^{\prime}}$ versus $L$ (multiplying by $k$ makes the results for different $q$ comparable) for three temperatures very near criticality. In Table II, we report the estimated dynamic

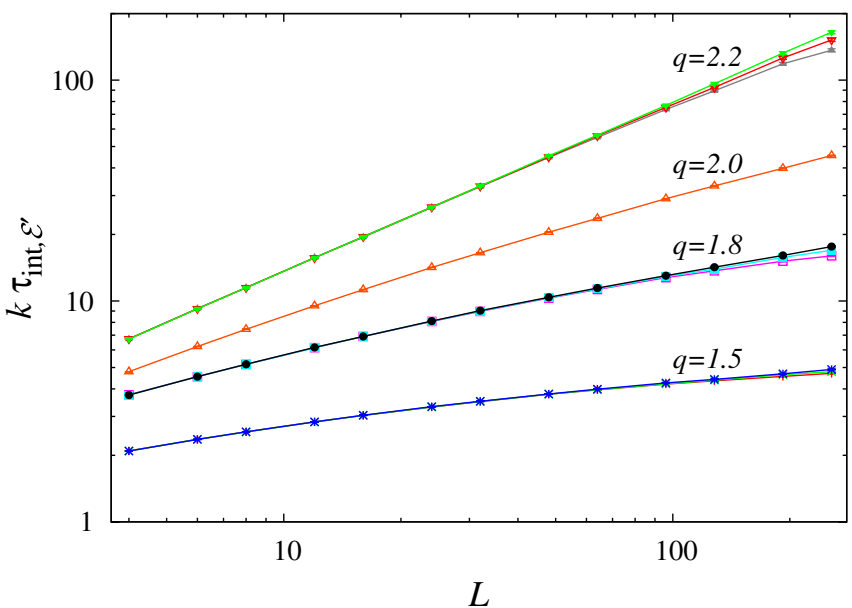

FIG. 3 (color online). $\quad k \tau_{\text {int, } \mathcal{E}^{\prime}}$ versus lattice size $L$ for ChayesMachta simulations of the three-dimensional random-cluster model with $q=1.5,1.8,2.2$ at three near-critical temperatures, taking $k=\lfloor q\rfloor$. Data for $q=2, k=2$ at approximate criticality are from Ref. [10].

critical exponents $z_{\text {int, } \mathcal{E}^{\prime}}$ and static critical exponents $\alpha / \nu$ and $\beta / \nu$. In Fig. 4 , we plot $k \tau_{\text {int, } \mathcal{E}^{\prime}} / C_{H}$ versus $L$. It seems clear that, for all four values of $q$, the Li-Sokal bound is far from sharp. On the other hand, from Table II, it seems clear that for $q=1.5,1.8$ we have the strict inequality $z_{\text {int, } \mathcal{E}^{\prime}}<$ $\beta / \nu$, once again ruling out the Ossola-Sokal conjecture even in its weak form.

The dynamic critical behavior of the SW-CM dynamic universality class in dimension $d=3$ therefore remains a mystery. Clearly, some new physical principle, beyond the slow equilibration of the energy embodied in the Li-Sokal bound [23], needs to be discovered.

One clue might be provided by our analysis [16] of the $\mathrm{CM}$ algorithm on the complete graph (mean-field limit), generalizing the analysis in Ref. [27] of the SW algorithm. Taking $k=1$ and defining a "magnetization" $m$ to be the fraction of sites in the largest cluster, we obtain for $1 \leq$ $q \leq 2$ the approximate difference equation [generalizing Eq. (10) in Ref. [27]]

$$
m^{\prime}=\frac{2 q-2}{q} m-\frac{4 t}{q^{2}}+\frac{8(q-1) t m}{3 q^{2}}-\frac{2(q-1)^{2} m^{2}}{3 q}
$$

TABLE II. Dynamic critical exponents $z_{\text {int, } \mathcal{E}^{\prime}}$ and static exponents $\alpha / \nu$ and $\beta / \nu$ for the three-dimensional random-cluster model. For $q=2$, dynamic data are from Ref. [10] and static exponents are from Ref. [26].

\begin{tabular}{lccccc}
\hline \hline$q$ & Fit & $L_{\min }$ & $z_{\text {int, } \mathcal{E}^{\prime}}$ & $\alpha / \nu$ & \multicolumn{1}{c}{$\beta / \nu$} \\
\hline 1.5 & $A L^{z}$ & 96 & $0.13(1)$ & $-0.32(4)$ & $0.500(4)$ \\
1.8 & $A L^{z}$ & 96 & $0.29(1)$ & $-0.15(5)$ & $0.5117(6)$ \\
2 & $A L^{z}$ & 96 & $0.46(3)$ & $0.174(1)$ & $0.5184(1)$ \\
2.2 & $A L^{z}$ & 24 & $0.76(1)$ & $0.50(4)$ & $0.508(4)$ \\
\hline \hline
\end{tabular}




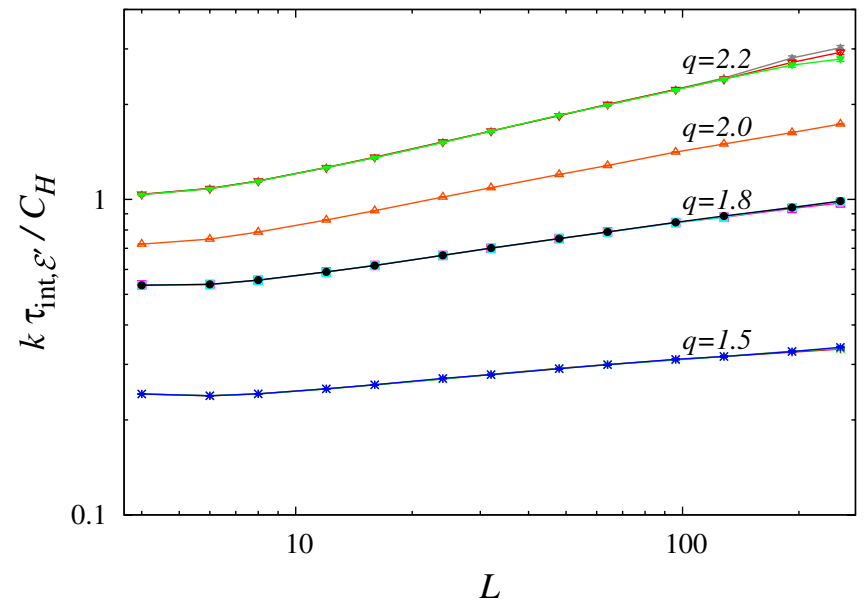

FIG. 4 (color online). $k \tau_{\text {int, } \mathcal{E}^{\prime}} / C_{H}$ versus lattice size $L$ for Chayes-Machta simulations of the near-critical threedimensional random-cluster model at $q=1.5,1.8,2,2.2$, with $k=\lfloor q\rfloor$. Data for $q=2$ at approximate criticality are from Ref. [10].

where $m^{\prime}$ is the value of $m$ after a sweep in which the active group contains the largest cluster, and $t$ is the deviation from the critical temperature. Clearly, $q=2$ is a special case because the coefficient of the linear term equals 1: we have $\beta=1 / 2$ and $z=1$, and it is clear from the derivations $[16,27]$ that $z$ is actually $\beta / \nu$. For $1 \leq q<2$, by contrast, both the statics and dynamics are in the percolation universality class with $\beta=1$ and $z=0$ : small perturbations from equilibrium relax exponentially with a finite autocorrelation time $\tau_{\text {exp }, m}=q / \log [q /(2 q-2)]$ that diverges as $q \uparrow 2$. We conjecture that a similar behavior holds above the upper critical dimension, which for $q<2$ is presumably $d=6$. Our numerical data [16] confirm the behavior $z=0$ for $1 \leq q<2$, with $\tau \propto 1 /(2-q)$ as $q \uparrow 2$, but not the predicted amplitude.

Details of these simulations and their data analysis will be reported separately $[15,16]$.

This work was supported in part by NSF Grants No. PHY-0116590 and No. PHY-0424082.

[1] Monte Carlo Methods in Statistical Physics, edited by K. Binder (Springer-Verlag, Berlin, 1986), 2nd ed.; Applications of the Monte Carlo Method in Statistical Physics, edited by K. Binder (Springer-Verlag, Berlin, 1987), 2nd ed.; The Monte Carlo Method in Condensed Matter Physics, edited by K. Binder (Springer-Verlag, Berlin, 1995), 2nd ed.

[2] I. Montvay and G. Münster, Quantum Fields on a Lattice (Cambridge University Press, New York, 1994), Chap. 7.

[3] P. C. Hohenberg and B. I. Halperin, Rev. Mod. Phys. 49, 435 (1977).

[4] A. D. Sokal, in Functional Integration: Basics and Applications, edited by C. de Witt-Morette, P. Cartier, and A. Folacci (Plenum, New York, 1997), pp. 131-192.
[5] R. H. Swendsen and J.-S. Wang, Phys. Rev. Lett. 58, 86 (1987).

[6] R. B. Potts, Proc. Cambridge Philos. Soc. 48, 106 (1952).

[7] F. Y. Wu, Rev. Mod. Phys. 54, 235 (1982); 55, 315(E) (1983); J. Appl. Phys. 55, 2421 (1984).

[8] P. W. Kasteleyn and C. M. Fortuin, J. Phys. Soc. Jpn. 26 (Suppl.), 11 (1969); C. M. Fortuin and P. W. Kasteleyn, Physica (Amsterdam) 57, 536 (1972).

[9] R. G. Edwards and A. D. Sokal, Phys. Rev. D 38, 2009 (1988).

[10] See G. Ossola and A. D. Sokal, Nucl. Phys. B691, 259 (2004) for a summary of the latest data.

[11] Second-order transitions occur also for the Ising $(q=2)$ model in dimensions $d>4$, but here the static behavior is mean-field. One expects the dynamic critical exponents likewise to be dimension-independent for $d \geq 4$ (with possible multiplicative logarithmic corrections at $d=4)$.

[12] L. Chayes and J. Machta, Physica (Amsterdam) 254A, 477 (1998).

[13] G. Grimmett, The Random-Cluster Model (SpringerVerlag, New York, 2006).

[14] We stress that $q_{c}(\mathcal{L})$ is not necessarily the same for all lattices of a given dimension $d$; the first-order or secondorder nature of the transition is a nonuniversal question. See H. W. J. Blöte, Y. Deng, X. Qian, and A. D. Sokal (to be published) for further discussion.

[15] T. M. Garoni, G. Ossola, M. Polin, and A. D. Sokal (to be published).

[16] Y. Deng, T. M. Garoni, J. Machta, and A. D. Sokal (to be published).

[17] D. Stauffer and A. Aharony, Introduction to Percolation Theory (Taylor \& Francis, London, 1992), 2nd ed.

[18] Y. Deng, T. M. Garoni, W. Guo, H. W. J. Blöte, and A. D. Sokal, Phys. Rev. Lett. 98, 120601 (2007).

[19] F. Y. Wu, J. Stat. Phys. 18, 115 (1978); A. D. Sokal, Comb. Probab. Comput. 10, 41 (2001), see Sect. 6.

[20] R.J. Baxter, Exactly Solved Models in Statistical Mechanics (Academic, London, 1982).

[21] N. Madras and A. D. Sokal, J. Stat. Phys. 50, 109 (1988), Appendix C.

[22] In $d=2$, the exact exponents are $\alpha / \nu=(4 g-12) / g$ and $\beta / \nu=(g-2)(6-g) / 8 g$, where $q=4 \cos ^{2}(\pi g / 4)$ and $2 \leq g \leq 4$. See, e.g., B. Nienhuis, J. Stat. Phys. 34, 731 (1984).

[23] X.-J. Li and A. D. Sokal, Phys. Rev. Lett. 63, 827 (1989); see also J. Salas and A. D. Sokal, J. Stat. Phys. 87, 1 (1997).

[24] The apparent failure of this bound in Table I for $q=4$ is presumably due to multiplicative logarithmic corrections [J. Salas and A. D. Sokal, J. Stat. Phys. 88, 567 (1997), Sect. 6]. Figure 2 shows that $\tau_{\text {int, } \mathcal{E}^{\prime}} / C_{H}$ is indeed bounded below.

[25] Y. Deng, T. M. Garoni, and A. D. Sokal, Phys. Rev. Lett. 98, 030602 (2007).

[26] A. Pelissetto and E. Vicari, Phys. Rep. 368, 549 (2002); Y. Deng and H.W.J. Blöte, Phys. Rev. E 68, 036125 (2003).

[27] N. Persky, R. Ben-Av, I. Kanter, and E. Domany, Phys. Rev. E 54, 2351 (1996). 